\title{
El consentimiento para tratamiento de datos personales de salud en tiempos del COVID-19
}

\author{
The consent for personal health data processing \\ in time of COVID-19
}

\author{
Raúl Vásquez Rodríguez ${ }^{[*]}$
}

\begin{abstract}
Resumen: el presente artículo se centra en la interacción entre los derechos fundamentales a la protección de los datos personales y a la protección de la salud, en el marco de la lucha contra el covid-19 en el Perú. Se inicia el estudio con el desarrollo constitucional de tales derechos, para luego revisar sus respectivas normas legales, teniendo como objetivo esclarecer una de las herramientas básicas que permiten superar los conflictos que se presenten entre ambos en la presente circunstancia de emergencia nacional por el covid-19, concerniente al consentimiento para el tratamiento de datos personales. Adicionalmente, se estudiarán dos casos de tratamiento de datos personales en acciones de prevención del covid-19, que evidencian la pacífica coexistencia entre los derechos constitucionales y los intereses surgidos de la actual situación sanitaria.
\end{abstract}

Palabras claves: protección de datos personales, datos de salud, covid-19.

Abstract: this paper focuses on interaction between fundamental rights of personal data protection and health protection, in the frame of fighting against covid-19 in Peru. This research begins with constitutional development of those rights, in order to review their related laws, having like an objective clarifying one of their basic legal resources which allow overcome any struggling between those rights during the current emergency state due to covid-19, related to consent for personal health data processing. In addition, two cases of personal data processing in preventing covid-19 actions will be studied, which show a peaceful interaction between aforementioned rights and interests arising from current emergency situation.

Keywords: personal data protection, health data, covid-19.

[*] Abogado y Magíster en Derecho de la Propiedad Intelectual y de la Competencia por la Pontificia Universidad Católica del Perú, con especialización en Derecho Administrativo por la Universidad de Salamanca. Miembro del Colegio de Abogados de Lima. Contacto: ravasquez78@yahoo.com 


\section{MARCO INTRODUCTORIO}

Es innegable la alteración que la pandemia del COVID-19 ha provocado en las actividades humanas y el desenvolvimiento de la sociedad, debido al desconocimiento de las formas de contrarrestar la enfermedad y sus agresivos síntomas, a su carácter asintomático que dificulta su detección y, sobre todo, a la alta probabilidad de transmisión o contagio de la misma, motivos por los cuales, a través del Decreto Supremo N. ${ }^{\circ}$ 044-2020-PCM, se declaró el estado de Emergencia Nacional y se adoptó una serie de medidas dirigidas a la prevención del contagio, tales como el aislamiento social, las restricciones de ciertos derechos fundamentales, como la libertad de reunión y movimiento; medidas que fueron complementadas a través de otras normas legales y lineamientos sectoriales.

Debe reconocerse que en una circunstancia como la actual, de grave riesgo para la salud pública y consecuente necesidad de prevención, la información relativa a la salud de las personas se hace imprescindible, lo que lleva a necesarios actos de recopilación y procesamientos con diversos fines, que van desde el diagnóstico hasta el rastreo o seguimiento de posibles focos de contagio, pasando por la preservación de condiciones de seguridad y salud mínimas para trabajadores. Se evidencia en tal situación que, para dar satisfacción a las mencionadas necesidades, será preciso manejar información personal de posibles infectados y personas de su entorno, significando ello una invasión a su privacidad.

Lo mencionado pone en manifiesto la interacción que existe entre el derecho a la salud, entendido ampliamente como derecho fundamental de la persona y como derecho constitucionalmente reconocido, y el específico derecho fundamental a la protección de datos personales, la cual, en las actuales circunstancias, haría ver en un primer momento un novísimo conflicto, de más difícil ponderación. No obstante, como iremos viendo, existen solucio- nes que permitan la concurrencia funcional de ambos derechos, surgidas de la Ley N. ${ }^{\circ} 29733$, Ley de Protección de Datos Personales (2011) (en adelante, LPDP) y su reglamento.

Para tal fin, se analizará preliminarmente los componentes normativos de cada uno de los derechos mencionados, comenzando por lo concerniente a la salud en general, para luego pasar al análisis del derecho fundamental a la protección de datos personales, a la normativa aplicable (LPDP y su reglamento), incidiendo en lo relativo al consentimiento para el tratamiento de los datos personales relativos a la salud, entendido este como la manifestación de voluntad consciente de la persona con la que controla el manejo de sus datos personales y por ende, como base de las garantías de licitud del tratamiento. Con ello, se irá trazando la forma en que el ordenamiento jurídico puede satisfacer ambos intereses, explicando la respuesta de la normativa a la actual situación de emergencia sanitaria y en general, a cualquier otro escenario de excepción donde tenga lugar la mencionada confrontación de derechos.

\section{EL DERECHO A LA PROTECCIÓN DE LA SALUD}

Comenzamos con la mención general del derecho, teniendo en consideración que este se debe entender derivado del derecho fundamental a la integridad, contemplado en el numeral 1 del artículo 2 de la Constitución Política del Perú de 1993 (en adelante, la Constitución), como uno de los bienes irrenunciables e inalienables que corresponden a la dignidad de la persona; y por otro lado, del derecho constitucionalmente protegido a la salud, previsto en el capítulo II del título I de la mencionada carta magna, compuesto de otras disposiciones referidas tanto a la salud individual, familiar y a la preservación de la misma en el ámbito de la comunidad (Rubio, 2017, pp. 5859), así como al manejo de recursos y a las obligaciones y garantías que debe prestar el Estado para el ejercicio de tales derechos. 
Cabe preguntarse cómo se podría hablar de un derecho fundamental a la salud, más allá de que se omita su mención expresa entre los numerales el artículo 2 de la Constitución. Para responder a tal inquietud, se debe descartar cualquier negativa a dicha posibilidad, tomando en cuenta lo establecido en el artículo 3 de la misma carta magna ${ }^{[1]}$, según la cual no existe óbice para el reconocimiento de derechos que deriven de la dignidad de la persona y que por ello, requieran de garantía constitucional, la cual corresponde a un factor inherente y esencial del desarrollo de la personalidad, como es la salud.

Por dicho carácter esencial, es que no es adecuado hablar de derechos «establecidos», término que se utiliza en el artículo 3 mencionado, sino del reconocimiento e individualización de los mismos (Rubio, Eguiguren y Bernales, 2010, pp. 797-800) como necesariamente existentes, producto de la evolución de los conceptos de los cuales surgen los derechos, y que pueden entenderse como autónomos respecto de alguno de los derechos fundamentales en los que inicialmente estarían implícitos. Esta situación encuentra un relevante desarrollo en sentencias del Tribunal Constitucional, como la recaída en el expediente $\mathrm{N}$. $^{\circ} 0008-2003 / \mathrm{Al}-\mathrm{TC}^{[2]}$.

\subsection{La composición del derecho fundamental a la salud}

Dejando de lado la terminología, puede entenderse el derecho a la integridad personal como aquel que incorpora a la salud como derecho fundamental de la persona, previsto en el numeral 1 del artículo 2 de la Constitución, conjuntamente a la vida, la identidad, así como al libre desarrollo y bienes. Tal ubicación sistémica, conjuntamente con otros valores, se debe a su carácter esencial para la construcción y defensa de la dignidad humana.

De acuerdo con Landa (2017), la integridad personal se compone tanto de la protección de la indemnidad somática o corporal de la persona frente a cualquier posible agresión o intervención no deseada, así de la indemnidad psíquica y moral, refiriéndose a la protección de la faceta emocional frente a perturbaciones tales como injurias o malos tratos en general, y en el caso de la indemnidad moral, a la protección de sus convicciones o creencias, entendidas como adhesiones racionalmente determinadas, con lo cual se añade al conjunto de hechos proscritos los ejecutados en entornos de represión política, como el trato inhumano o degradante y la tortura (Canosa, 2017, pp. 264-266). Dichas facetas de la integridad son manifestaciones del libre desarrollo de la personalidad, que requieren una protección reforzada de parte del Estado y mediante el ordenamiento jurídico.

Como derecho subjetivo, surge el derecho y deber de la persona de preservar su propia integridad en las tres facetas vistas anteriormente, lo cual la posibilita a repeler cualquier intervención siempre que esta sea no deseada o no necesaria, mientras que le impide también atentar contra su propia anatomía o psiquis, siendo la regla la imposibilidad de disponer de su integridad (Rubio, Eguiguren y Bernales,

[1] «Artículo $3^{\circ}$. La enumeración de los derechos establecidos en este capítulo no excluye los demás que la Constitución garantiza, ni otros de naturaleza análoga o que se fundan en la dignidad del hombre, o en los principios de soberanía del pueblo, del Estado democrático de derecho y de la forma republicana de gobierno.» (Const., 1993, art. 3)

[2] «32. (...) Es de verse que en la Constitución existe una pluralidad de casos referidos a ciertos atributos que, siendo genéricos en su naturaleza, y admitiendo manifestaciones objetivamente incorporadas en el mismo texto fundamental, suponen un numerus apertus a otras expresiones sucedáneas.

Así, el artículo 3 de la Constitución prevé la individualización de 'nuevos' derechos, en función de la aplicación de la teoría de los 'derechos innominados', allí expuesta y sustentada.» (Exp. N. . 0008-2003/Al-TC, 2003) 
2010, pp. 117-119). Es necesario resaltar el carácter no absoluto de este derecho-deber, que se hace tangible en casos en los que tales intervenciones se deben a una necesidad de salud, como el caso de los actos médicos, u obedezcan a la voluntad de la persona, como en casos humanitarios que se aprecian en las donaciones de órganos, donde puede disponer de su integridad física.

Por su parte, del mismo derecho fundamental emanan deberes para el Estado en su calidad de garante de las libertades y derechos fundamentales que sustentan el estado de Derecho (Esteve, 2015, p. 13) y terceros particulares. El más elemental de ellos es evitar cualquier intervención que lesione la integridad de la persona, principalmente cuando esta no obedezca a su voluntad o sea necesaria para evitarle un perjuicio mayor. Derivado de él, surge otro deber del Estado, en su papel de garante de los derechos y de la dignidad humana, el cual puede subdividirse en dos: El de investigar y sancionar las mencionadas lesiones, y adoptar las acciones pertinentes para prevenir o evitar afectaciones a la integridad.

Entonces, queda establecido que existe, como derecho fundamental, la conservación del bienestar corporal y psíquico de las personas, así como el deber de garante del Estado para con el mismo, con el cual se da pie a uno de los derechos sociales constitucionalmente reconocidos: El derecho a la salud, desarrollado en diversos artículos del capítulo II del mismo título de la Constitución.
En primer término, el artículo 7 de nuestra carta magna ${ }^{[3]}$ concibe a la salud como un bien de interés tanto para el individuo, su entorno más íntimo (la familia) y su entorno exterior (trabajo, actividades, otras relaciones), el cual se compone tanto de las comunidades a la que pertenece y que conforman la sociedad en general. Para dichos tres estamentos, existe la obligación de protección, promoción y defensa de la salud, a cargo del Estado, de forma directa e indirecta, vale decir, a través de la prestación de los servicios asistenciales y de la dirección en la forma de dicha prestación.

Lo concerniente a dicha obligación halla su sustento en el artículo 9 de la Constitución ${ }^{[4]}$, en el que se establece al Estado como responsable de la política nacional de salud y al Poder Ejecutivo como supervisor de su diseño, conducción y cumplimiento, siendo garante del acceso equitativo a los servicios de salud. Ello implica una labor continua en diversos aspectos relacionados con dichos servicios, referidos a su planificación, ejecución y supervisión de cualquier programa dirigido a la preservación de la integridad de las personas y la salud pública; labor a cargo de entidades del Poder Ejecutivo.

El mencionado rol de garante de la prestación los servicios de salud, es establecido en el artículo 11 de la Constitución ${ }^{[5]}$, artículo que muestra al Estado en dos facetas: Como principal diseñador de los programas generales a nivel nacional, así como confirma lo mencionado respecto de su rol como prestador directo de

[3] «Artículo $7^{\circ}$. Todos tienen derecho a la protección de su salud, la del medio familiar y la de la comunidad así como el deber de contribuir a su promoción y defensa. (...)».

(Const., 1993, art. 7)

[4] «Artículo 9. El Estado determina la política nacional de salud. El Poder Ejecutivo norma y supervisa su aplicación. Es responsable de diseñarla y conducirla en forma plural y descentralizadora para facilitar a todos el acceso equitativo a los servicios de salud».

(Const., 1993, art. 9)

[5] «Artículo $11^{\circ}$. El Estado garantiza el libre acceso a prestaciones de salud y a pensiones, a través de entidades públicas, privadas o mixtas. Supervisa asimismo su eficaz funcionamiento».

(Const., 1993, art. 11) 
dichos servicios, por un lado, y el de regulador y fiscalizador de su adecuada prestación por parte de entidades privadas; situaciones en las que se concreta el contenido del derecho y su condición de interés público: Preservar la mejora del nivel de vida de las personas, garantizando el acceso equitativos y disponibilidad de servicios de salud adecuados. (Landa, 2017, p. 161)

Lo descrito anteriormente demuestra la existencia de un derecho fundamental a la protección de la salud, en virtud del cual se demanda diversas acciones a cargo del Estado, dirigidas proteger a la persona en su integridad, las cuales pueden incluir incluso la limitación de otros derechos constitucionales, debido a su trascendencia. Por supuesto, estas acciones son desarrolladas en normativa infraconstitucional, específicamente a través de la Ley N . $^{\circ}$ 26842, Ley General de Salud (1997) (en adelante, LGS), en la forma que se verá a continuación.

\subsection{Desarrollo legal del derecho a la protec- ción de la salud}

La protección de constitucional que recibe la salud, como condición indispensable para el desarrollo humano, hace, a su vez, que esta adquiera la condición de interés público y por ello, necesario el desarrollo legal de su protección. Es a través de la LGS que se establecen los derechos, deberes, restricciones y responsabilidades que se deben asumir para una adecuada prestación de servicios de salud, así como las disposiciones generales concernientes al derecho a la protección de la salud, previstas estas en su Título Preliminar.

El artículo II de dicho título de la LGS, se reafirma la calidad de interés público que tiene la protección a la salud, de la que consecuentemente se deriva el deber del Estado consistente en su vigilancia, regulación y promoción. Dicha situación implica, como ya se anotó, poner en práctica labores de fiscalización, planificación, así como el diseño de programas generales y para situaciones particulares, y su continua supervisión.
Involucrada con esa responsabilidad general, se encuentra la específica del artículo IV, referida con la conducción de la salud pública, vinculada con la prestación y programas de salud aplicables a toda la población, que incorpora en su dinámica la protección de la salud individual, cuya responsabilidad el Estado comparte con la sociedad y el individuo. De este artículo, se desprende el rol de comando del Estado en cuanto a las políticas y disposiciones que atañen a los servicios y procesos generales de protección de la salud, a través de normas que son de orden público, como establece el artículo IX del mismo título, correspondiendo a su alta relevancia.

Ahora bien, es conveniente tener en cuenta, dentro de los artículos del Título Preliminar, uno específico al manejo de la información en salud por parte de la autoridad nacional de salud, el artículo XIV, que establece la obligación de las personas la información concerniente a la salud que sea exigible, según ley. Esta disposición, junto con la cualidad de norma de orden público que ostentan las normas referidas a salud, demuestran la efectiva fuerza normativa que la recopilación de información puede tener para limitar ciertos derechos fundamentales (que tampoco son absolutos) en determinadas circunstancias, como sucede con los derechos relacionados a la intimidad, la privacidad y protección de datos personales, los cuales se estudiarán en el siguiente apartado.

\section{DERECHO FUNDAMENTAL A LA PROTEC- CIÓN DE LOS DATOS PERSONALES}

Para estudiar este derecho fundamental, es necesario entender su autonomía de otras facetas de lo que en su origen, principalmente en el derecho anglosajón, se tiene por right to be alone, consistente en la soberanía de todo hombre sobre su propio espacio, sin importar cuál sea su condición (Saldaña, 2012, p. 204), desarrollado en un primer momento por el juez de la Suprema Corte del Estado de Michigan Thomas Cooley en 1879 y por Louis Brandeis y Samuel Warren en su artículo «The Right of Privacy» de 1890. 
El entendimiento unidimensional del mencionado derecho de la persona se mantuvo en el ordenamiento jurídico peruano con la Constitución Política del Perú de 1979, en cuyo artículo $2^{[6]}$ se adoptó una redacción omnicomprensiva que comprendía a la intimidad personal y familiar como referente general al resguardo de dos niveles de espacio personal, junto al honor y a la reputación. De ello, se desprende un derecho a la protección de la información vinculada a tales niveles de intimidad, consistente en el poder de repeler intromisiones por parte de terceros, sin desarrollar otras prerrogativas respecto de la información de la persona.

La disgregación de los derechos fundamentales de la persona sobre su espacio se verá en la siguiente Constitución peruana, en cuyo artículo 2 se tienen dos numerales ${ }^{[7]}$, uno referido al honor, reputación, voz, imagen e intimidad, y uno novedoso respecto de la información correspondiente a las personas en sus ámbitos personal y familiar, fortaleciendo las facultades que ellas tienen sobre tal información, siendo titulares de esta.

\subsection{Derecho fundamental a la autodetermi- nación informativa o a la protección de datos personales}

En el texto constitucional, se aprecia que los numerales 6 y 7 de su artículo 2, incluyen como objeto de protección a la intimidad personal y familiar, pudiendo resultar vana la separación de ambos derechos fundamentales, a simple vista. Sin embargo, es preciso determinar las garantías que cada una contiene, de acuerdo con sus respectivos textos, lo cual es decisivo para demarcar el camino de la presente investigación; para ello, resulta conveniente comenzar por el numeral 7 señalado.

Dicho numeral ofrece, como garantía para la intimidad personal y familiar, la prohibición del escudriñamiento y/o divulgación no deseada de los actos de las personas, en su esfera totalmente individual, como en aquellas más próximas en las que se desenvuelve (familiar, amical, laboral); teniendo como objetivo inmediato la inviolabilidad de tales actos, y como objetivo mediato el equilibrio necesario para su desarrollo individual y en sociedad (Fernández, 2004, p. 59). Con ello, se establece una prohibición general de intromisión, un impedimento para acciones de terceros respecto de la información del sujeto, como su acceso a esta o su extracción.

Por su parte, en el texto del numeral 6 de dicho artículo constitucional se aprecia la protección contra el suministro de información correspondiente a una determinada persona, que pueda afectar su intimidad personal y familiar (Zegarra, 2011), y en general, siguiendo el desarrollo jurisprudencial que analizaremos en breve, contra acciones ejercidas por terceros respecto de tal información, lo que hace que la integridad de su derecho dependa del comportamiento de aquellos, pudiendo ser controlado de acuerdo con la voluntad o el criterio del titular de la información.

[6] «Artículo 2. Toda persona tiene derecho:

(...)

5. Al honor y la buena reputación, a la intimidad personal y familiar y a la propia imagen (...)». (Const., 1979, art. 2, núm. 5)

[7] «Artículo $2^{\circ}$. Toda persona tiene derecho:

(...)

6. A que los servicios informáticos, computarizados o no, públicos o privados, no suministren informaciones que afecten la intimidad personal y familiar.

7. Al honor y a la buena reputación, a la intimidad personal y familiar así como a la voz y a la imagen propias.» (Const., 1993, art. 2, núm. 6 y 7) 
En tal sentido, se aprecia una importante diferencia conceptual respecto del derecho contemplado en el numeral 7, pues al consistir en el control de comportamientos ajenos, premune a la persona de la facultad de ejercer señorío sobre su información y de determinar qué se hace con ella (Lete, 1996, pp. 176-177). Con ello, no solo premune a la persona del poder de repeler intromisiones, sino también de prevenir, evitar y/o revertir los efectos de actividades de terceros que expongan su información personal, se tenga o no su consentimiento u otro supuesto que legitime tal tratamiento de información.

Es así que el derecho fundamental a la protección de datos personales, denominado también «autodeterminación informativa» en la jurisprudencia del Tribunal Constitucional, confirma su distinción, al permitir controlar cualquier acto de tratamiento de su información personal mediante las facultades que le otorga a la persona (Castro, 2008, pp. 261-262), con lo cual se satisface la necesidad de equilibrio a favor del individuo en el actual contexto de circulación de información (Pérez, 1996, pp. 23-24), de acelerada evolución de las tecnologías mediante las cuales se da tal circulación y del uso predominante de estas por parte de entidades públicas y privadas que ejercen poderes fácticos, ya sea en el gobierno o en el mercado, o inclusive de particulares cuyo empoderamiento reside en el manejo de considerable cantidad de información, el denominado «poder informático», que implica el traslado de buena porción del conflicto a la esfera de los privados (Sagüés, 1998, p. 189).

Los caracteres del derecho fundamental mencionado son reconocidos en el Perú a través de sentencias del Tribunal Constitucional como la recaída en el expediente $N$. $^{\circ}$ 1797-2002$\mathrm{HD}^{[8]}$, que reconoce su autonomía respecto del derecho a la intimidad contemplado en el numeral 7 , gracias a los poderes que otorga sobre terceros. Este último aspecto es reforzado en la sentencia del expediente $\mathrm{N} .^{\circ}$ 4739-2007-PHD/ $\mathrm{TC}^{[9]}$, en la que se desarrolla la facultad de ejercer tal derecho fundamental ante el accionar de personas naturales o jurídicas, públicas o privadas, confirmando el imperio del titular sobre su información personal, por medio del control de las acciones de terceros sobre tal acervo informativo, cuyo ejercicio dependen de la voluntad consciente de la persona respecto del manejo de dicha información.

Por su parte, debe señalarse que tal derecho subjetivo otorgado a la persona no sería del todo funcional si no emanaran de este derecho fundamental deberes $u$ obligaciones hacia los terceros que realizan la manipulación o tratamiento de los datos personales, así como deberes del Estado mismo para implementar un sistema de protección (Landa,2017, pp. 7677). Dichos deberes u obligaciones, así como el desarrollo de principios rectores de los que depende la licitud del tratamiento de los datos personales, se encuentran desarrollados en la LPDP y su reglamento.

[8] «3. (...) el derecho a la autodeterminación informativa no puede identificarse con el derecho a la intimidad, personal o familiar, reconocido, a su vez, por el inciso 7) del mismo artículo $2^{\circ}$ de la Constitución. Ello se debe a que mientras que este protege el derecho a la vida privada, esto es, el poder jurídico de rechazar intromisiones ilegítimas en la vida íntima o familiar de las personas, aquel garantiza la facultad de todo individuo de poder preservarla controlando el registro, uso y revelación de los datos que les conciernen.» (Exp. N. ${ }^{\circ}$ 1797-2002/HD, 2003)

[9] «2. El derecho a la autodeterminación informativa consiste en la serie de facultades que tiene toda persona para ejercer control sobre la información personal que le concierne, contenida en registros ya sean públicos, privados o informáticos, a fin de enfrentar las posibles extralimitaciones de los mismos. Se encuentra estrechamente ligado a un control sobre la información, como una autodeterminación de la vida íntima, de la esfera personal.» (Exp. N. $\left.{ }^{\circ} 4739-2007 / \mathrm{HD}, 2007\right)$ 


\subsection{Desarrollo legal y reglamentario del de- recho fundamental a la protección de da- tos personales}

La LPDP y su reglamento son las normas mediante las cuales se desarrollan las prerrogativas de las personas, que componen el derecho fundamental a la protección de datos personales, tales como los derechos denominados «ARCO» que se ejercen sobre el contenido de los registros de su información, así como sobre las acciones de tratamiento. Por otro lado, se establecen las condiciones de licitud de tratamiento, cimentadas en sus principios rectores, de las cuales emanan las obligaciones a cumplir por las personas o entidades que efectúen dicho tratamiento, las cuales se encaminan también a tutelar los derechos de las personas titulares de los datos personales.

Es preciso tomar en cuenta también que la tutela de tales derechos, así como la fiscalización del cumplimiento de las obligaciones de quienes son responsables de efectuar el tratamiento, se desarrolla por medio de procedimientos e instancias que dichas normas establecen (Landa, 2017, p. 77) y encomiendan a la Autoridad Nacional de Protección de Datos Personales diversas competencias y funciones para brindar efectivamente dicha tutela, en instancia administrativa.

En este punto del trabajo, conociendo la generalidad de lo dispuesto por la norma encaminada a garantizar el derecho a la protección de la salud y por el otro lado, la que desarrolla el derecho fundamental a la protección de datos personales, corresponde analizar cómo es que a través de las disposiciones de esta última, más precisamente las concernientes al consentimiento, se consigue el equilibrio en el ejercicio de ambos derechos, en circunstancias que favorecen su conflicto como el de la presente emergencia sanitaria, a fin de convertirlo en una interacción que proteja los derechos e intereses de las personas.

\section{TRATAMIENTO DE LOS DATOS PERSO- NALES EN EL ÁMBITO DE LA SALUD, DE ACUERDO CON LA NORMATIVA DE PRO- TECCIÓN DE DATOS PERSONALES}

Al regular todo lo concerniente al tratamiento de datos personales, la LPDP cumple con determinar no solamente su ámbito subjetivo u objetivo, más bien, en este último, define los elementos que se involucran con dicha acción, con la finalidad de permitir su mejor conocimiento y distinción de otras figuras, acciones o cosas, y entender el sentido de las disposiciones sobre ellos. Con dicho propósito esclarecedor, es que se ahondará a continuación en las definiciones más relevantes, por tratarse de los más básicos y por tener una mayor incidencia que otros.

El artículo 2 de la LPDP contiene una lista de tales definiciones, entre las cuales se aprecia la de «tratamiento de datos personales» en su numeral $19^{[10]}$, que consiste en una lista de actividades, aisladas o incorporadas a procedimientos, que se realizan con datos personales, tales como la recopilación, el almacenamiento, la organización, el bloqueo, la supresión, así como diversas modalidades de traslado de dominio de los datos, como puede ser la transferencia por comunicación a destinatarios

[10] «Artículo 2. Definiciones

Para todos los efectos de la presente ley, se entiende por:

(...)

19. Tratamiento de datos personales. Cualquier operación o procedimiento técnico, automatizado o no, que permite la recopilación, registro, organización, almacenamiento, conservación, elaboración, modificación, extracción, consulta, utilización, bloqueo, supresión, comunicación por transferencia o por difusión o cualquier otra forma de procesamiento que facilite el acceso, correlación o interconexión de los datos personales».

(Ley 29733, 2011, art. 2, núm. 19) 
específicos o por difusión. Dicha lista es enunciativa, permitiendo entender como tratamiento a cualquier acción ejercida con los datos, conocida o por conocer.

Asimismo, se aprecia que en el numeral 4 de dicho artículo, se define de forma general los datos personales como «información sobre una persona natural que la identifica o la hace identificable a través de medios que pueden ser razonablemente utilizados», debiendo entender una doble dimensión del concepto: Por un lado, como un dato vinculado únicamente con una persona (su titular) que permite identificarla plenamente, como sucede con el número de DNI, el cual es asignado a un solo individuo; y, de otra parte, comprende en sí los datos que, sin tener un grado tan alto de vinculación con la persona, brinda alcances sobre ella, informando sobre imagen o apariencia, datos de contacto personal, información laboral, crediticia, de hábitos o preferencias, entre otros datos vinculados a la persona.

Ahora bien, es preciso tener en cuenta que la precitada definición es un género, dentro del cual se encuentra una especie de datos personales, los denominados «datos sensibles». El numeral 5 del artículo 2 de la LPDP define como tales a una clase de datos personales relacionados con la esfera más íntima de las personas, tales como la salud, la filiación política, sindical o la vida sexual, entre otros; datos cuyo tratamiento ilícito o inadecuado implica un mayor riesgo para los derechos y libertades de la persona, como posibles actos de estigmatización o hechos discriminatorios (Remolina, 2013, p. 149).

La segregación de esta clase de datos tiene un efecto práctico en la normativa de protección de datos personales: Sujeta el tratamiento de dichos datos personales a condiciones más estrictas, tales como mayores obligaciones y sanciones más graves en caso de su incumplimiento, obedeciendo al mayor riesgo para la persona que conlleva un tratamiento inadecuado o ilícito. En el siguiente apartado, se estu- diará lo referido al principio de consentimiento de la LPDP, al ser uno de los supuestos de legitimidad más importantes del tratamiento de datos personales de salud (en su calidad de datos sensibles), verificando cómo es que se aplican tales disposiciones a casos que tienen lugar en el Perú con motivo de la pandemia por el COVID-19.

Acerca de los datos personales de salud, es importante tener en cuenta entender tal concepto de forma amplia, considerando en sí cualquier información que pueda relacionarse a su salud física y psíquica. Con ello, se puede entender como un dato personal de salud no solo lo que subyace al acto médico, sino que incluye exámenes, informaciones preliminares (síntomas, historias clínicas), y otros de índole no «médica», que permitan establecer antecedentes o pronósticos de la persona, como información del entorno familiar o social, siendo un concepto que no debe entenderse de forma restrictiva (Di Pizzo, 2018, pp. 249-250) datos entregados por la misma persona, a través de dispositivos electrónicos, como elementos del internet de las cosas o aplicaciones móviles (Álvarez, 2016, p. 174).

\subsection{Contenido del principio de legalidad de la LPDP, como presupuesto de licitud del tratamiento}

En la LPDP se tiene una lista enunciativa de principios aplicables al tratamiento de los datos personales, a través de los cuales se establecen las pautas a las que debe ajustarse todo tratamiento de datos personales (Zamudio, 2012, p. 45), y que sirven también como raíces y fuentes interpretativas de las disposiciones subyacentes en dicha ley, al precisar sus finalidades generales. En tal sentido, es válido pensar en los principios como supuestos cuyo cumplimiento legitima el tratamiento, supuestos que establecen las características fundamentales de su licitud, así como en su función informadora e integradora respecto de otros artículos (Palma, 2018, p. 40). 
En el artículo 4 de la LPDP se encuentra el principio de legalidad ${ }^{[11]}$, según el cual, el tratamiento de datos personales debe ejecutarse en el marco de medios y relaciones jurídicas lícitas, respaldadas por el ordenamiento jurídico lícita (Puyol, 2016, p. 141), que no impliquen la comisión de un delito, infracción administrativa y/o incumplimiento de índole civil, proscribiéndose acciones como la recopilación basada en el fraude, la coacción o cualquier tratamiento desleal o fraudulento (Castro, 2008, p. 269). A su vez, este principio también dispone que el tratamiento deberá someterse a las disposiciones de la misma ley, por lo que su cumplimiento no será autoevaluativo, si no que dependerá del acatamiento de las disposiciones mencionadas, así como de otras normas. Entonces, el cumplimiento de este principio rector se sustenta con el cumplimiento de los otros principios y de las disposiciones que emanen de cada uno, como el principio de consentimiento.

\subsection{Contenido del principio de consentimiento de la LPDP}

El principio de consentimiento del artículo 5 de la LPDP ${ }^{[12]}$ constituye uno de los supuestos fundamentales de legitimidad de tratamiento de datos personales, así como uno de los primeros en el tiempo conjuntamente con el deber de información, dentro de cualquier proceso de tratamiento. A través de dicho artículo se establece, de forma sucinta, la acción a la cual está obligado el responsable del mencionado tratamiento en situaciones ordinarias: Obtener la manifestación de voluntad favorable por parte del titular de los datos personales, la cual es producto de la «autodeterminación informativa» mediante la cual, el titular de los datos personales decide si permite o no el tratamiento, siguiendo unas condiciones mínimas de conocimiento y libertad.

Ahora bien, dichas condiciones constituyen supuestos del cumplimiento del principio mencionado y se estructuran por medio de otros artículos de la misma ley y su reglamento, como el inciso 13.5 del artículo $13^{[13]}$ de la ley y el artículo 12 de dicho reglamento; los mismos que hacen válida la manifestación del consentimiento por parte de la persona que es titular de los datos, cuyo cumplimiento corre por cuenta del responsable del tratamiento:

- Libre: La manifestación de voluntad de otorgar el consentimiento (como todo acto jurídico contemplado en el Código Civil) no debe someterse a coacción, engaño, dolo o cualquier otra distorsión o impedimento de sus facultades; evitándose también la extensión del consentimiento a tratamientos no reconocibles por el titular de los datos personales (Trujillo, 2018, p. 55).

- Previo: El consentimiento debe otorgarse antes del inicio de la acción de tratamiento para la cual se solicita.

[11] «Artículo 4. Principio de legalidad

El tratamiento de los datos personales se hace conforme a lo establecido en la ley. Se prohíbe la recopilación de los datos personales por medios fraudulentos, desleales o ilícitos».

(Ley 29733, 2011, art. 4)

[12] «Artículo 5. Principio de consentimiento

Para el tratamiento de los datos personales debe mediar el consentimiento de su titular».

(Ley 29733, 2011, art. 5)

[13] «Artículo 13. Alcances sobre el tratamiento de datos personales

(...)

13.5 Los datos personales solo pueden ser objeto de tratamiento con consentimiento de su titular, salvo ley autoritativa al respecto. El consentimiento debe ser previo, informado, expreso e inequívoco».

(Ley 29733, 2011, art. 13, inc. 13.5) 
- Expreso e inequívoco: El consentimiento debe manifestarse de forma tangible, ya sea de forma escrita (virtual o física), verbal o a través de una conducta indiscutiblemente favorable a autorizar el tratamiento.

- Informado: Para obtener el consentimiento, el responsable debe otorgar, de forma previa, la información detallada sobre factores básicos del tratamiento a realizar y las condiciones en las que se efectuará (siguiendo lo establecido en el numeral 4 del artículo 12 y el artículo 18 de la LPDP); permitiendo al titular de los datos personales su pleno entendimiento y con ello, la formación de su voluntad consciente.

Como se señaló al inicio de este capítulo, en el caso del tratamiento de datos sensibles, existen obligaciones mayores para el responsable en este caso, mediante las cuales se pretende dar una mayor garantía de la certeza con la que el titular de tales datos manifiesta su voluntad. Por ello, de acuerdo con lo establecido en el inciso 13.6 del artículo 13 de la LPDP ${ }^{[14]}$ y el artículo 14 de su reglamento, exigiéndose que el consentimiento se otorgue por escrito, con la finalidad de tener un documento que sustente su obtención válida (Adsuara, 2016, p. 160).

No obstante, debe tomarse en cuenta otro factor importante previsto en el inciso 13.6 del artículo 13 mencionado, referente a la posibilidad de que el tratamiento se efectúe sin el consentimiento del titular de los datos personales, en caso exista una autorización legal que atienda a razones de interés público; cuestión importante que obedece al carácter no absoluto del derecho fundamental, pero que circunscribe las posibilidades de su limitación a lo dispuesto por una norma legal y a situaciones de interés público. Desde ese punto, corresponde analizar las situaciones excepcionales en las que no se requiere el consentimiento para el tratamiento de datos personales sensibles, contempladas en el artículo 14 de la LPDP.

\subsection{Excepciones a la obligatoriedad del con- sentimiento para el tratamiento de datos personales}

El artículo 14 de la LPDP ${ }^{[15]}$ contiene un listado de supuestos que pueden aceptarse como legitimadores per se del tratamiento,

[14] «Artículo 13. Alcances sobre el tratamiento de datos personales

(...)

13.6 En el caso de datos sensibles, el consentimiento para efectos de su tratamiento, además, debe efectuarse por escrito. Aun cuando no mediara el consentimiento del titular, el tratamiento de datos sensibles puede efectuarse cuando la ley lo autorice, siempre que ello atienda a motivos importantes de interés público».

(Ley 29733, 2011, art. 13, inc. 13.6)

[15] «Artículo 14. Limitaciones al consentimiento para el tratamiento de datos personales

No se requiere el consentimiento del titular de datos personales, para los efectos de su tratamiento, en los siguientes casos:

1. Cuando los datos personales se recopilen o transfieran para el ejercicio de las funciones de las entidades públicas en el ámbito de sus competencias.

(...)

5. Cuando los datos personales sean necesarios para la preparación, celebración y ejecución de una relación contractual en la que el titular de datos personales sea parte, o cuando se trate de datos personales que deriven de una relación científica o profesional del titular y sean necesarios para su desarrollo o cumplimiento.

6. Cuando se trate de datos personales relativos a la salud y sea necesario, en circunstancia de riesgo, para la prevención, diagnóstico y tratamiento médico o quirúrgico del titular, siempre que dicho tratamiento sea realizado en establecimientos de salud o por profesionales en ciencias de la salud, observando el secreto profesional; o cuando medien razones de interés público previstas por ley o cuando deban 
al punto de permitir su realización sin el consentimiento del titular de los datos personales, hecho que implica la limitación del derecho fundamental a la protección de datos personales, delatando su carácter no absoluto. Dicha prescindencia tiene su origen en causales que reemplazan a la voluntad consciente de la persona, verificadas en los siguientes escenarios:

- Cuando la voluntad de consentir del tratamiento se manifiesta a través de otro acto que, para su concreción, requiere de tal tratamiento, como el establecimiento de una relación contractual (comercial o laboral) por parte del titular o un acto en beneficio de los intereses del mismo, que no se puede realizar sin sus datos personales.

- Cuando el tratamiento de datos personales es permitido para el cumplimiento de una norma con rango de ley, que representa los intereses o necesidades de la nación; o para satisfacer un interés público relevante calificado como tal por el Poder Ejecutivo, donde el derecho individual cede ante el bien común, como sucede respecto de un derecho constitucional a la protección de la salud y la salud pública, con el menor impacto posible para la persona.

- Cuando el tratamiento es necesario para el ejercicio de competencias atribuidas a una entidad pública.
Es necesario tener en cuenta que pese a la especial protección que la normativa brinda a los datos sensibles, el tratamiento de estos no escapa a estas situaciones que prescinden del consentimiento, en virtud de los escenarios descritos y por una situación que surge de la redacción legal: El artículo mencionado no hace distinción entre las clases de datos personales objeto de tratamiento, porque en esos casos, se entenderá que hay un interés superior que justifica esta flexibilidad en las exigencias.

Dicha situación encuentra sustento por diversos pronunciamientos de la Autoridad Nacional de Protección de Datos Personales, por medio de diversos oficios de absolución de consulta, en los que interpreta de forma extensiva la aplicación de las mencionadas excepciones, sin importar el tipo de datos personales objeto de tratamiento[16].

Entendido ello, se debe anotar en primer lugar el numeral 1 del artículo 14 de la LPDP, referido a dos actividades específicas de tratamiento de datos personales, recopilación y transferencia, efectuadas por las entidades públicas en el ámbito de sus competencias. Al respecto, debe tenerse en cuenta que tales competencias no serían solamente las contempladas en las normas de organización y funciones que tienen algunos ministerios ni las contenidas en leyes orgánicas y de creación de entidades; más bien, tales competencias

tratarse por razones de salud pública, ambas razones deben ser calificadas como tales por el Ministerio de Salud; o para la realización de estudios epidemiológicos o análogos, en tanto se apliquen procedimientos de disociación adecuados.

(...)»

(Ley 29733, 2011, art. 13, num. 1, 5 y 6)

[16] Al respecto, conviene revisión a los siguientes oficios de absolución de consulta:

Oficio N. ${ }^{\circ}$ 240-2013-JUS-DGPDP

Recuperado de: https://www.minjus.gob.pe/wp-content/uploads/2014/07/2.-Datos-personales-relacionadoscon-la-salud.pdf

Oficio N. ${ }^{\circ}$ 247-2014-JUS-DGPDP

Recuperado de: https://www.minjus.gob.pe/wp-content/uploads/2014/07/4.-Datos-sensibles-y-limitacionesal-consentimiento.pdf

Oficio N. ${ }^{\circ}$ 570-2015-JUS-DGPDP

Recuperado de: https://www.minjus.gob.pe/wp-content/uploads/2015/12/570-II.pdf 
pueden estar enmarcadas también en leyes sectoriales, como sucede con la LGS, como norma rectora de la prestación de los servicios de salud.

Corresponde hacer referencia, en segundo lugar, a la excepción más recurrente para las actividades de tratamiento, contenida en el numeral 5 del artículo 14 de la LPDP, la cual centra su justificación en la necesidad de los datos personales para los actos preparatorios, celebración, ejecución y cumplimiento de un contrato, sea este de índole civil o laboral.

Tal situación implica que los datos personales son relevantes para asegurar la continuidad de las prestaciones y contraprestaciones a cargo de los partes, lo cual puede evidenciarse con el uso de una aplicación móvil, que es descargada por un usuario que brinda datos personales que son necesarios para activar la función deseada por este, como sucede con una evaluación de estado físico o su ubicación, cuya carencia impide que el usuario, como cliente de la empresa que desarrolla dicha aplicación, obtenga el beneficio esperado.

En lo específico de los casos laborales, también se aprecia la necesidad del tratamiento de datos personales en el cumplimiento de la relación laboral, ya sea en la fiscalización a cargo del empleador y en el cumplimiento de las obligaciones que le competen, así como en la adopción de condiciones mínimas necesarias para el desempeño del trabajador, como sucede con cuestiones de seguridad y salud en el trabajo.

A continuación, corresponde revisar un escenario normativo específicamente dirigido al tratamiento de datos personales en el ámbito de la salud, contemplado en el numeral 6 del mismo artículo. Debe entenderse que dicha excepción cubre tres situaciones con alcance distinto, que son las siguientes:

- El tratamiento encaminado a la prevención, diagnóstico, tratamiento médico o quirúrgico del titular o en circunstancia de riesgo, realizándose en un establecimiento de salud o por un profesional de la salud; escenario donde prevalece la vida y la salud del individuo por sobre su voluntad, para lo cual son útiles los datos de salud de la persona.

- Razones de interés público previstas en una ley y declaradas como tales por el Poder Ejecutivo en las que prevalece el bien público establecido por norma con rango de ley sobre la voluntad del individuo, lo cual se hace mucho más evidente en casos de salud pública ante amenazas transfronterizas graves (Trujillo,2018, p. 67).

- Análisis epidemiológicos o procedimientos análogos, debiendo procederse con la disociación de los datos personales.

Es pertinente la diferenciación de estos tres supuestos, puesto que, siguiendo la redacción del numeral, solo el primero de ellos estaría exclusivamente circunscrito a los datos personales de salud, lo cual obedece a su naturaleza centralmente asistencial, concerniente a los actos médicos que se realizan sobre una persona. Los otros supuestos obedecen a casos de índole más general, encaminados a la elaboración de políticas o medidas sanitarias de prevención, detección o erradicación de enfermedades, las cuales requieren de mayores elementos informativos que la información estrictamente médica, como sucede con la ubicación, información sobre servicios y vivienda, entre otros que permitan analizar condiciones sociales o económicas.

\section{CASOS EN LOS QUE SE APLICAN EXCEP- CIONES A LA OBLIGACIÓN DE OBTENER EL CONSENTIMIENTO PARA EL TRATAMIEN- TO DE DATOS PERSONALES, SURGIDOS DURANTE EL ESTADO DE EMERGENCIA}

Desde el 16 de marzo, se decretó a nivel nacional el Estado de Emergencia con miras a enfrentar los estragos del virus COVID-19, cuyo grado de extensión fue declarado previamente por la Organización Mundial de la Salud como pandemia. En tal sentido, mediante el Decreto Supremo N. ${ }^{\circ}$ 044-2020-PCM, se dispuso en su 
artículo 1 la medida de aislamiento social para todos los ciudadanos, así como la suspensión de derechos como la libertad de tránsito y de reunión, al prohibirse eventos que conlleven aglomeraciones sociales, con la finalidad de impedir o reducir al mínimo la posibilidad de contagio.

En tales circunstancias, teniendo como finalidad de recabar información sobre zonas de mayor incidencia de contagio y brindar a los ciudadanos un pronóstico sobre su posible condición de portador del COVID-19, la Presidencia del Consejo de Ministros lanzó la aplicación móvil «El Perú en tus Manos», que ofrece la opción «Mapa de zonas afectadas» (figura 1) para acceder a un mapa de cercanías a él donde se marca la incidencia de contagios; así también, se dispone de la opción «Triaje» (figura 2) mediante el cual, de acuerdo con los síntomas que consigne, podrá determinar si es o no un posible portador del mencionado virus. Como se puede apreciar, en el uso de la aplicación móvil señalada se efectúa el tratamiento de datos personales, incluyendo los de salud, cuya responsabilidad recae en el Ministerio de Salud, en su calidad de autoridad nacional de salud de acuerdo con la LGS.

\section{Figura 1}

Opción «Mapa de zonas afectadas» de la aplicación «El Perú en sus manos»

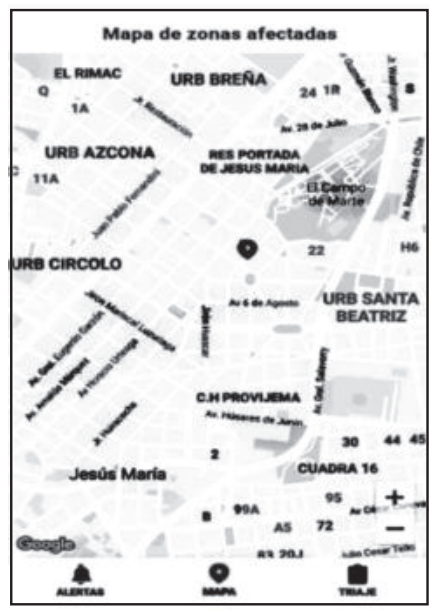

Figura 2

Opción «Triaje» de la aplicación «El Perú en sus manos»

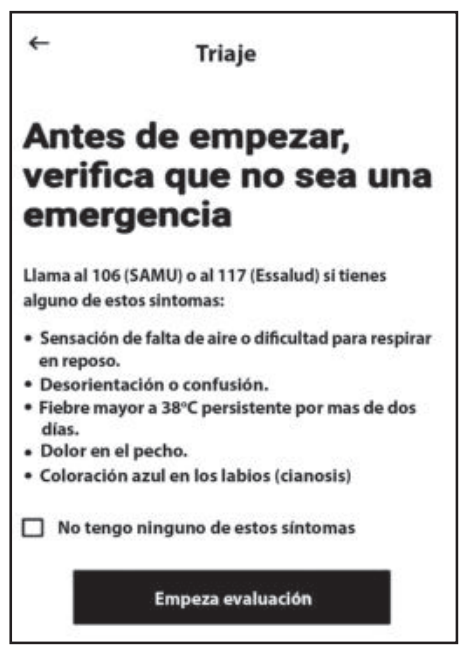

De otro lado, aún durante la vigencia de las medidas de aislamiento social, y con el fin de responder a la necesidad de reinicio de actividades laborales paralizadas por seguridad sanitaria, el Ministerio de Salud emitió la Resolución Ministerial N. ${ }^{\circ}$ 239-2020-MINSA, mediante el cual se aprobaron los «Lineamientos de vigilancia para la salud de los trabajadores con riesgo de exposición a COVID-19»[17] siendo el segundo de ellos (punto 7.2.2) el referido a la evaluación del estado de salud de los trabajadores de las empresas, previa al retorno a los centros de trabajo. Como en el caso anterior, es apreciable el tratamiento de datos de salud de trabajadores que, siguiendo tales lineamientos, se realizaría.

Ambos casos sirven de ejemplo para verificar como a través de las disposiciones referidas al consentimiento del tratamiento de datos personales, se encuentra un punto de concordia entre los derechos cuya interacción se analiza en este trabajo. Conviene señalar que dicha concordia es facilitada también por

[17] Véase: https://cdn.www.gob.pe/uploads/document/file/668359/RM_239-2020-MINSA_Y_ANEXO.PDF 
el límite impuesto por el principio de legalidad del Título Preliminar de la Ley del Procedimiento Administrativo General ${ }^{[18]}$, que demanda la juridicidad o concordancia de toda actuación de las entidades del estado con el ordenamiento jurídico general, especialmente con las normas legales, como manifestación de la voluntad popular a la que se hayan subordinadas tales entidades (Martín, 1967, p. 66); con lo que en el caso analizado, tenemos las disposiciones de LPDP como uno de los límites impuestos a los procedimientos encaminados a enfrentar el COVID-19.

\subsection{Aplicación móvil «El Perú en tus manos»}

En el caso de la mencionada aplicación móvil, como ya se indicó, existen dos funcionalidades interesantes, cada una de las cuales conlleva una actividad de tratamiento de datos personales distinta, cuyas legitimidades se determinan, cada cual, de forma autónoma. Sin embargo, de forma previa a ello, lo correcto es señalar que para la activación de la aplicación, se utilizan los datos personales identificativos del usuario (nombres, DNI) así como sus datos de geolocalización, obtenidos del GPS de cada dispositivo móvil.

Ahora bien, para obtener los resultados deseados de la función de triaje de la aplicación móvil, también es preciso que el usuario preste sus datos personales de salud, como son los síntomas que tiene en ese momento, con los cuales, podrá obtener el diagnóstico preliminar que lo orientará a decidir por mantener las medidas de seguridad que normalmente lleva o reforzarlas.

Entonces, se aprecian dos supuestos en los cuales las mencionadas actividades de tra- tamiento estarían exentas de la obligación de obtener el consentimiento:

- El tratamiento de los datos personales en cuestiones relativas a la salud: Este supuesto de tratamiento de los datos personales de salud conjuntamente con los datos de geolocalización, se encuadra en el segundo supuesto de excepción del numeral 6 del artículo 14 de la LPDP, pues implica una medida de ubicación geográfica de posibles focos de contagio (que se aprecia en la opción «Mapa de zonas afectadas»), de la cual solo se muestra al usuario de la aplicación un acercamiento a su zona más cercana, con la finalidad de que adopte las medidas preventivas de contagio que considere necesarias.

Así también, esta información es utilizada en el seguimiento del avance geográfico de la enfermedad, lo cual se involucra con el tercer supuesto del mencionado numeral, siempre que la información sea disociada o anonimizada, desvinculada de la persona a la que corresponde, utilizándose como cifra numérica para el estudio de la epidemia,

El necesario tratamiento de datos personales para la funcionalidad esperada por el usuario: Esta situación, correspondiente al supuesto del numeral 5 del artículo 14 de la LPDP, se evidencia en que para que el usuario obtenga los resultados que busca, debe aportar sus datos personales, tanto los de salud (para determinar el diagnóstico en la opción «Triaje») como los de geolocalización (a fin de obtener su ubicación y relacionarla con el estado de sus cercanías).

[18] «Artículo IV. Principios del procedimiento administrativo

1. El procedimiento administrativo se sustenta fundamentalmente en los siguientes principios, sin perjuicio de la vigencia de otros principios generales del Derecho Administrativo:

1.1. Principio de legalidad. - Las autoridades administrativas deben actuar con respeto a la Constitución, la ley y al derecho, dentro de las facultades que le estén atribuidas y de acuerdo con los fines para los que les fueron conferidas.»

(Ley del Procedimiento Administrativo General, Título Preliminar, art. IV, núm. 1) 
En cuanto al uso de los datos personales de salud para la opción «Triaje», es discutible la aplicación del numeral 6 del mencionado artículo, toda vez que, si bien su uso se encamina al diagnóstico, se desconoce si es realizado o no por un profesional de la salud. No obstante, encaja en los otros dos supuestos, al ser información utilizada en políticas y estudios epidemiológicos relacionados con la prevención y control del COVID-19, que es un asunto declarado de emergencia nacional cuyo control está a cargo del Ministerio de Salud como autoridad de salud de nivel nacional, de acuerdo con el artículo 76 de la LGS ${ }^{[19]}$.

\section{2. "Lineamientos de vigilancia para la salud de los trabajadores con riesgo de exposi- ción a COVID-19», aprobados por la Reso- lución Ministerial N. 239-2020-MINSA}

El punto 7.2.2 de los lineamientos aprobados por la autoridad nacional de salud, el Ministerio de Salud, se refiere a la evaluación que se la hace a los trabajadores que retornan a sus puestos de trabajo, con la finalidad de descartar que sean portadores del COVID-19. En tal sentido, el responsable de seguridad y salud de cada empresa debe aplicar la Ficha Sintomatológica COVID-19 del Ministerio de Salud, a cada empleado y, de acuerdo con las respuestas que se ofrezcan sobre síntomas, ejercitar otras pruebas, como la toma de temperatura; con los sospechosos de contagio, son sometidos a otras evaluaciones, así como a la aplicación de la Ficha Epidemiológica COVID-19.

Resulta evidente, por la finalidad de los lineamientos, que la práctica de pruebas y eva- luaciones de síntomas a los empleados que retornan a los centros de trabajo, buscan que los empleadores utilicen estas herramientas para asegurar condiciones mínimas de salubridad y de mínimo riesgo de contagio del COVID-19. En tal sentido, la recopilación de los datos personales de salud de sus trabajadores se relaciona con el escenario previsto en el numeral 5 del artículo 14 de la LPDP, puesto que, dentro del ámbito laboral, las pruebas de salud señaladas tienen como objetivo la detección de un presunto infectado y la toma de las medidas pertinentes para evitar el contagio en otros trabajadores.

Ahora bien, si revisamos el subnumeral 7.2.2.2, correspondiente a las medidas a tomar en caso de detectarse un resultado positivo para COVID-19 en un trabajador, aparte del llenado de la Ficha Epidemiológica COVID-19, se contempla la obligación de identificar a los contactos domiciliarios de dicho trabajador, así como el respectivo reporte a la autoridad de salud. En este caso, se aprecia que en el tratamiento de datos personales (incluyendo identificativos y de salud del trabajador, familiares y/o cohabitantes) hay una intervención de la mencionada autoridad, amparada justamente en el articulado de la LGS que tiene como finalidad la realización de estudios epidemiológicos, necesarios para sus labores de prevención y contención de la pandemia, reconocida como una situación de interés público, debiendo proceder con la disociación de tal información.

No debe obviarse que fuera de las disposiciones de la LGS, este tratamiento de datos personales encuentra amparo en la Ley $\mathrm{N} .^{\circ} 29783$, Ley de Seguridad y Salud en el Trabajo (2012), precisamente a partir del Principio del Prevención

[19] «Artículo 76.- La Autoridad de Salud de nivel nacional es responsable de dirigir y normar las acciones destinadas a evitar la propagación y lograr el control y erradicación de las enfermedades transmisibles en todo el territorio nacional, ejerciendo la vigilancia epidemiológica e inteligencia sanitaria y dictando las disposiciones correspondientes.

Así mismo tiene la potestad de promover y coordinar con personas e instituciones públicas o privadas la realización de actividades en el campo epidemiológico y sanitario».

(Ley 26842, 1997, art. 76) 
del artículo I de su Título Preliminar[20], que obliga a los empleadores a adoptar todas las medidas necesarias para proteger la vida, la salud y bienestar de los trabajadores, evitando riesgos de contagio, como los que se presentan en el caso de la pandemia del COVID-19.

De tal manera, se configuran el segundo y el tercer supuesto del numeral 6 y el del numeral 1 del artículo 14 de la LPDP, como bases de la no obligatoriedad del consentimiento para las acciones de tratamiento descritas, llevadas a cabo por la autoridad nacional de salud.

\section{CONCLUSIONES}

El carácter no absoluto de los derechos fundamentales se hace tangible en el marco de la interacción (no «conflicto») entre la protección de la salud y la de los datos personales, como la que se presenta en una circunstancia de excepción por emergencia sanitaria en el Perú debida al COVID-19, en la cual es necesario incluso el manejo de datos personales sensibles de las personas.

En tal sentido, para atender las necesidades de salud no solo de la persona, sino de toda la comunidad ante la amenaza que representa para la salud pública el COVID-19, es imprescindible la relativización del derecho a la protección de datos personales. Dicha relativización se aprecia respecto del consentimiento, como manifestación de voluntad consciente de la persona y como arma de control sobre el tratamiento que pueda efectuar un tercero con sus datos personales; puesto que en un escenario como el planteado, se sustrae al consentimiento la calidad de principal elemento de legitimación de tal tratamiento, en razón de otros supuestos válidos que reemplazan a la voluntad consciente del titular de los datos personales, en el rol legitimador.

Ahora bien, es preciso señalar que dichos supuestos legitimadores actúan en el ordenamiento como supuestos excepcionales y no como regla, y deben estar establecidas en normas de rango legal, de acuerdo con las disposiciones de la LPDP que ya se estudiaron. En razón a ello, dicha ley, en su artículo 14, establece tales supuestos excepcionales, que representan situaciones más relevantes que la voluntad consciente de la persona para permitir el tratamiento de sus datos personales, como las concernientes al interés público, al desempeño de las competencias de una entidad pública establecidas por ley, o a la manifestación de voluntad de la misma persona cuando esta se dirige al desarrollo de una actividad que necesita del tratamiento de sus datos personales.

En el caso de la situación de emergencia debida al brote del COVID-19 en nuestro país, se han configurado diversas situaciones legitimadoras del tratamiento que exceptúan la obligación de obtener el consentimiento, que convergen en los tres escenarios explicados y que se encuentran previstas en los numerales 1,5 y 6 del artículo 14 de la LPDP, al encajar en tales supuestos de hecho normados.

Por supuesto, en la LPDP existen otras obligaciones que los responsables del tratamiento, como son las empresas empleadoras y la autoridad nacional de salud, deben acatar,

[20] «Título Preliminar

Principios

I. Principio de prevención

El empleador garantiza, en el centro de trabajo, el establecimiento de los medios y condiciones que protejan la vida, la salud y el bienestar de los trabajadores, y de aquellos que, no teniendo vínculo laboral, prestan servicios o se encuentran dentro del ámbito del centro de labores. Debe considerar factores sociales, laborales y biológicos, diferenciados en función del sexo, incorporando la dimensión de género en la evaluación y prevención de los riesgos en la salud laboral.»

(Ley 29783, 2011, art. I) 
a fin asegurar la confidencialidad y la recopilación no excesiva ni invasiva de datos personales, garantizando el respeto a la privacidad e intimidad de las personas. No se debe olvidar que la no obligatoriedad del consentimiento, en los supuestos estudiados, no dispensa a los responsables del tratamiento de cumplir con sus otras obligaciones, durante el desarrollo del proceso de tratamiento, el cual es cíclico, de múltiples etapas.

En virtud de ello, se puede considerar que el ordenamiento jurídico peruano garantiza una interrelación equilibrada entre los derechos mencionados, que no deja desprotegidos los intereses que por ambos lados se busca satisfacer; garantía que se consigue con el acatamiento de las normas de la LPDP durante el tratamiento, así como el cese de la mencionada relativización de la protección de los datos personales con el cese del estado de excepción en el que se desenvuelve la materia de este estudio.

\section{REFERENCIAS}

Adsuara, B. (2016). El Consentimiento. En Piñar Mañas, José Luis (Dir.). Reglamento General de Protección de Datos. Hacia un Nuevo Modelo Europeo de Privacidad. Madrid: Editorial Reus, S.A.

Álvarez, C. (2016). Tratamiento de datos de salud. En Piñar Mañas, José Luis (Dir.). Reglamento General de Protección de Datos. Hacia un Nuevo Modelo Europeo de Privacidad. Madrid: Editorial Reus, S.A.

Canosa, R. (2017). La protección de la integridad personal. Revista de Derecho Político. N. ${ }^{\circ}$ 100, pp. 257-310. http://revistas. uned.es/index.php/derechopolitico/article/ view/20700/17175

Castro, K. (2008). El Derecho Fundamental a la Protección de Datos Personales: Aportes para su Desarrollo en el Perú». Ius et Veritas, vol. 18 (N. $\left.{ }^{\circ} 37\right)$, pp. 260-277.

Coz, D. (2015). El Principio de Finalidad en la Recopilación y Tratamiento de Datos Persona- les. Diálogo con la Jurisprudencia, N. ${ }^{\circ} 201$, pp. 33-36.

Dávara, I. (2011). Hacia la Estandarización de la Protección de Datos Personales. Madrid: Editorial La Ley.

Dávara, M. Á. (2015). Manual de Derecho Informático. Navarra: Editorial Aranzadi S.A.

Di Pizzo, A. (2018). La Expansión del Derecho al Olvido Digital. Efectos de «Google Spain» y el Big Data e implicaciones del Nuevo Reglamento Europeo de Protección de Datos. Barcelona: Editorial Atelier.

Esteve, J. (2015). La administración garante. Una aproximación. Revista de Administración Pública, N. ${ }^{\circ}$ 197, pp. 11-39. Doi: http:// dx.doi.org/10.18042/cepc/rap. 197.01

Fernández, C. (2004). Derecho de las Personas. Exposición de Motivos y Comentarios al Libro Primero del Código Civil Peruano. Lima: Grijley.

Landa, C. (2017). Los derechos fundamentales. Lima: Pontificia Universidad Católica del Perú, Fondo Editorial.

Lete, J. M. (1996). Derecho de la Persona. Madrid: Editorial Tecnos, S.A.

Lorenzo, S. (2018). Posición Jurídica de los Intervinientes en el Tratamiento de Datos Personales. En Murga Fernández, Juan Pablo, Fernández Scagliusi, María de los Ángeles y Espejo Lerdo de Tejada, Manuel (Dir.). Protección de Datos, Responsabilidad Activa y Técnicas de Garantía. Madrid: Editorial Reus, S.A.

Martín, R. (1967). «La Sustantividad del Derecho Administrativo». Revista de la Administración Pública N. ${ }^{\circ}$ 53, 1967, pp. 35-72. Recuperado de: https://dialnet.unirioja.es/descarga/articulo/2116255.pdf

Morón, D. (2017). Comentarios a la Ley del Procedimiento Administrativo General. Lima: Gaceta Jurídica S.A.

Palma, A. (2018). «Principios Relativos al Tratamiento de Datos Personales». En Murga 
Fernández, Juan Pablo, Fernández Scagliusi, María de los Ángeles y Espejo Lerdo de Tejada, Manuel (Dir.). Protección de Datos, Responsabilidad Activa y Técnicas de Garantía. Madrid: Editorial Reus, S.A.

Pérez, A. E. (1996). Manual de Informática y Derecho. Barcelona: Editorial Ariel

Puyol, J. (2016). Los Principios del Derecho a la Protección de Datos. En Piñar Mañas, José Luis (Dir.). Reglamento General de Protección de Datos. Hacia un Nuevo Modelo Europeo de Privacidad. Madrid: Editorial Reus, S.A.

Remolina, N. (2013). Tratamiento de Datos Personales: Una Aproximación Internacional y Comentarios a la Ley 1581 de 2012. Bogotá: Editorial Legis S.A.

Rubio, M. (2017). Para conocer la Constitución de 1993. Lima: Pontificia Universidad Católica del Perú, Fondo Editorial.

Rubio, M., Eguiguren, F. y Bernales, E. (2010). Los Derechos Fundamentales en la Jurisprudencia del Tribunal Constitucional. Análisis de los artículos 1, 2 y 3 de la Constitución. Lima: Pontificia Universidad Católica del Perú, Fondo Editorial.

Sagüés, N. P. (1998). Habeas Data: Su desarrollo constitucional. En $V$ Congreso Iberoamericano de Derecho Constitucional. Instituto de Investigaciones Jurídicas - Universidad Nacional Autónoma de México, Serie: G, Estudios Doctrinales, N. ${ }^{\circ} 193$, pp. 859-872. https://archivos.juridicas.unam.mx/www/ bjv/libros/1/113/39.pdf

Saldaña, M. N. (2012). Right to privacy: La génesis de la protección de la privacidad en el sistema constitucional norteamericano: El centenario legado de Warren y Brandeis». UNED Revista de Derecho Político. Madrid, N. ${ }^{\circ} 85$, septiembre-diciembre 2012, pp. 195240. http://rabida.uhu.es/dspace/bitstream/ handle/10272/7015/The_right_to_privacy. pdf? sequence $=2$
Trujillo, C. (2018). Las Basas de Legitimación del Tratamiento de Datos Personales. En Especial, el Consentimiento. En Murga Fernández, Juan Pablo, Fernández Scagliusi, María de los Ángeles y Espejo Lerdo de Tejada, Manuel (Dir.). Protección de Datos, Responsabilidad Activa y Técnicas de Garantía. Madrid: Editorial Reus, S.A.

Zamudio, M. (2012). El marco normativo latinoamericano y la ley de protección de datos personales del Perú. Revista Internacional de Protección de Datos Personales. Bogotá, Julio-diciembre 2012 (N. $\left.{ }^{\circ} 1\right)$, p. 2-21.

Zegarra, D. (2011). La Ley de Protección de Datos Personales, Ley 29733. Enfoque Derecho. https://www.enfoquederecho.com/2011 /08/04/la-ley-de-proteccion-de-datos-personales-ley-2973/

\section{REFERENCIAS JURÍDICAS}

Constitución Política del Perú, (1979). http://www. pcm.gob.pe/wp-content/uploads/2013/09/ Constitucion-Pol\%C3\%ADtica-del-Peru-1993.pdf

Ley de Protección de Datos Personales. [N. ${ }^{\circ}$ 29733] (3 de julio de 2011). Lima-Perú; Diario oficial El Peruano. http://www.leyes.congreso.gob.pe/Documentos/Leyes/29733.pdf

Ley General de Salud. [N. ${ }^{\circ}$ 26842] (9 de julio de 1997). Lima-Perú, Diario oficial El Peruano. http://www.essalud.gob.pe/transparencia/ pdf/publicacion/ley26842.pdf

Ley de Seguridad y Salud en el Trabajo. [N. ${ }^{\circ}$ 29783] (19 de agosto del 2011). Lima-Perú Diario oficial El Peruano. https://www.minagri.gob.pe/portal/download/pdf/seguridady-salud/reglamento-ley29783.pdf

Texto Único ordenado de la Ley del Procedimiento Administrativo General [N. $\left.{ }^{\circ} 27444\right]$, aprobado por Decreto Supremo N. ${ }^{\circ}$ 004-2019JUS. [25 de enero de 2019], Diario oficial El 
Peruano. http://www.pcm.gob.pe/wp-content/uploads/2013/09/Ley-de-Procedimiento-Administrativo-de-PersonalLey27444.pdf

Sentencia N. ${ }^{\circ}$ 0008-2003/Al-TC. (2003). https:// www.tc.gob.pe/jurisprudencia/2003/000082003-Al.html
Sentencia N. ${ }^{\circ}$ 1797-2002/HD. (2003). https:// www.tc.gob.pe/jurisprudencia/2003/017972002-HD.html

Sentenciada N. ${ }^{\circ}$ 4739-2007/HD. (2017). https:// www.tc.gob.pe/jurisprudencia/2008/047392007-HD.pdf 